\title{
Impact of Match Results on the Physical Performance of Players in Japanese Elite Rugby Union Match Play
}

\author{
Hayato Yamamoto $^{1 *}$, Masanori Takemura ${ }^{2}$, Misato Tachibana ${ }^{1}$, Junzo Tsujita $^{3}$ and Tatsuya Hojo ${ }^{1}$ \\ 'Graduate School of Health and Sports Science, Doshisha University, 1-3 Tataramiyakodani, Kyotanabe, Kyoto 610-0394 Japan \\ E-mail: trainer_life_22@yahoo.co.jp \\ ${ }^{2}$ Ichihashi clinic, 5-7-15 Fukaekitamachi, Higashinada-ku, Kobe, Hyogo 658-0013 Japan \\ ${ }^{3}$ Institute of Health and Sports Medicine Science, 2-14 Uedahigashimachi, Nishinomiya, Hyogo 663-8133 Japan
}

[Received January 20, 2020; Accepted May 15, 2020; Published online May 30, 2020]

\begin{abstract}
Purpose: The purpose of this study was to analyze the impact of match results (win-loss, score differential) on the physical performance of rugby union teams to improve training and conditioning and provide ideas for use on the playing field.

Methods: Data were obtained from 45 official matches over the 2013, 2014, and 2015 seasons of one team. Global positioning system units with built-in accelerometers were worn by the players; the per-match total distances, accelerations, and impact forces were calculated for the forwards and backs. Match results consisted of two elements: win-loss result and score differential.

Results: Data showed that each parameter had high values in closely fought matches when a team lost or when the point differential was small. In addition, physical performance was impacted differently from one position to another. Forwards engaged in significantly less high-acceleration and high-impact activity in wins but significantly higher activities in close matches $(\boldsymbol{p}<\mathbf{0 . 0 5})$. Backs engaged in significantly higher impacts (i.e., harder/more tackles) in close matches $(p<0.05)$.

Conclusions: Based on these data, some suggestions for new approaches that can be adopted by strength and conditioning coaches to optimize training-related performance and prevent sports injuries have been included.
\end{abstract}

Key words: match analysis, performance, global positioning system, coaching

\section{Introduction}

Rugby union differs in physical performances required during match depending on position (Duthie et al., 2003, Fuller et al., 2007, Yamamoto et al., 2020). It involves 10 positions for 15 players, and representative position groups are classified as forwards and backs. The characteristics are significantly different between the forwards and backs positions. Therefore, it is necessary to evaluate each position when analyzing physical performances.

GPS units with built-in accelerometers are commonly used to analyze in-match physical performance in team sports. Attributes of physical performance, such as the distance and speed of travel among players in a large number of sports, including rugby union (Austin et al., 2011), rugby league (Kempton et al., 2014), Australian Rules football (Wisbey et al., 2010),
Gaelic football (Reilly et al., 2015), soccer (Mallo et al., 2015), and hockey (Jennings et al., 2012), have been assessed.

In a rugby union, McLaren et al. (Rugby Football Union Championship) (McLaren et al., 2016), Kenneth et al. (the New Zealand All Blacks) (Quarrie et al., 2013), and Yamamoto et al. (Japan Rugby Top League) (Yamamoto et al., 2017) have reported the results of such studies. Interestingly, the total distance traveled in a match was different for each study: 5400-5960 m (RFU Championship), 5400-6300 m (All Blacks), and 5604-7001 m (Japan Rugby Top League). Thus, even with the same sport, differences in play between leagues and teams can be discerned. In a similar manner, differences in other performance indicators can be observed.

Physical performance in matches is influenced by a 
variety of factors, including win-loss result, score differential, opposition, strategy, and environmental factors; of these, the influence of win-loss result, score differential, and opposition on physical performance was analyzed (Gabbett, 2013, Rampinini et al., 2007, Sullivan et al., 2014). However, while it is expected that match results influence physical performance similarly in rugby union, no study about such notion has been published. Validating the impact of match results on physical performance allows for a detailed analysis of physical performance data, and the knowledge can then be used to identify the unique characteristics of each position and create position-specific training programs designed to improve performance. Thus, the purpose of this study was to shed light on the impact of match results (win-loss, score differential) on the physical performance of rugby union teams and apply the knowledge gained to the playing field.

\section{Methods}

\subsection{Experimental approach to the problem}

This study analyzed the relationship between matchday physical performance and match results (win-loss, score differential) in the context of an elite male rugby union team, specifically a team in the Japan Rugby Top League. Statistical comparisons were used to determine these potential relationships and whether such relationships were influenced by position.

\subsection{Participants}

The participants were the 15 starting team members in each match for one team belonging to the top league. The team played 14 matches in 2013 (4-10 win-loss record), 16 matches in 2014 (6-10), and 15 matches in 2015 (4-10-1), with a total of 45 matches (14-30-1) over the three seasons. For the purposes of this study, the participant population included only those players who played at least one full half. Players who were substituted during the first or second half were excluded from the sample. The total sample size was 838 .

This study was approved by the ethics committee of the Doshisha University (approval number: 17084). The objectives and possible limitations of the study were explained to the potential subjects, and informed consent was obtained from the participants prior to data collection.

\subsection{Global positioning system analysis}

This study used GPS units (SPI Pro X; GPSports Systems, Canberra, Australia) with built-in accelerometers with sampling frequencies of 15 and $100 \mathrm{~Hz}$. The GPS-unit was placed on the upper middle back between the scapulae of the subject using special protective vest recommended by the manufacturer. The physical performance parameters were as follows: (1) distance traveled, (2) acceleration, and (3) impact, as described below.

\subsubsection{Distance traveled}

The distance traveled in each of the 40-min first and second halves was calculated as the total distance (TD). In addition, data about distance were stratified by three speed zones (SZ): SZ-1, low velocity running $(0-12.0 \mathrm{~km} / \mathrm{h}) ; \mathrm{SZ}-2$, cruising $(12.1-18.0 \mathrm{~km} / \mathrm{h})$; and SZ-3, high velocity running $(>18.1 \mathrm{~km} / \mathrm{h})$.

\subsubsection{Acceleration}

Acceleration was calculated based on the GPS data as velocity per unit time $\left(\mathrm{m} / \mathrm{s}^{2}\right)$ and was stratified into three zones: acceleration zone (AZ)-1 (low acceleration), $<1.50 \mathrm{~m} / \mathrm{s}^{2} ;$ AZ-2 (moderate acceleration), 1.51$2.50 \mathrm{~m} / \mathrm{s}^{2}$; and AZ-3 (high acceleration), $>2.50 \mathrm{~m} / \mathrm{s}^{2}$.

\subsubsection{Impact}

Impact was calculated based on the accelerometer data. The three-dimensional (triaxial) linear accelerometric values were collected and converted into gravity $\mathrm{g}$-force values by dividing the raw value by 9.81 $\mathrm{m} / \mathrm{s}^{2}$. Impact was classified as moderate (IZ-1, 8.1-10 g) and high (IZ-2, > $10 \mathrm{~g})$ impact zones.

\subsection{Match results data collection}

Match results included win-loss result and score differential. Win-loss was scored as a binary value (i.e., no ties), and score differential was binned according to small (seven points or lower) and large (eight points or higher) difference categories. Match results were collected from the Top League official website.

\subsection{Statistical analyses}

Data were presented as mean \pm standard deviation. Bartlett's test was used to test the homoscedasticity of variances, and Welch's t-test was used to determine statistical significance in terms of total distance trav- 
eled, acceleration, and impact for each forwards and backs. Statistical significance was set at $\mathrm{p}<0.05$. Effect size was calculated using Cohen's d. Effect sizes of $<0.19,0.20-0.49,0.50-0.79$, and $>0.80$ were considered trival, small, medium, and large, respectively.

\section{Results}

Table 1 shows the relationship between physical performance and win-loss. Forwards obtained significantly higher values for IZ-1 and IZ-2 in losses relative to wins. For backs, the SZ-2, total acceleration (TA), and IZ-2 values were significantly higher in losses relative to wins.

Table 2 shows the relationship between physical performance and score differential. Forwards obtained significantly higher values for TD, TA, AZ-1, AZ-2, and IZ-2 in small score differentials relative to large ones; meanwhile, backs had significantly higher values for SZ-1 and IZ-2 in small differentials relative to large ones.

\section{Discussion}

The purpose of this study was to validate the impact of match results on the physical performance of a rugby union team. It only included data obtained from players who played the full first or second half because extrapolating data for a 40 -min half for players who were substituted during a half leads to decreased accuracy. In addition, this study considered the relationship between match results (win-loss, score differential) and physical performance separately in each 40-min half. Analyzing data on a player playing a full 80-min match produced large biases in the win-loss record. Because dividing a match into 40-minute halves lead to a reduction in biases in the win-loss record, this study considered the relationship between physical performance and match results (win-loss, score differential) in each half separately.

For analysis in this study, player positions were classified as backs or forwards rather than by individual position. Analysis was not performed by position, as has been reported in similar research in sports, such as Australian Rules football and rugby league (Gabbett, 2013, Sullivan et al., 2014). However, it is considered that there are distinctive relationships between physical performance and match results according to position. Furthermore, such distinctive positional relationships can be reflected in training programs; therefore, this study classified players as forwards or backs.

When considering the results of physical performance analysis according to win or loss, forwards were significantly higher in IZ-1 and IZ-2 and backs in SZ-2, TA, and IZ-2 in losses relative to wins. In fact, subjects obtained higher values across every parameter in the event of a loss. In the forwards, differentials of 15 times (win: 69 times; loss: 84 times) and

Table 1 Impact of win and loss on physical performances in elite japanese rugby union match play.

\begin{tabular}{|c|c|c|c|c|c|c|c|}
\hline & \multirow[b]{2}{*}{ Unit } & \multicolumn{3}{|c|}{ Forwards } & \multicolumn{3}{|c|}{ Backs } \\
\hline & & $\begin{array}{c}\text { Win } \\
(\mathrm{n}=152)\end{array}$ & $\begin{array}{c}\text { Loss } \\
(\mathrm{n}=219)\end{array}$ & $\begin{array}{l}\text { Effect size } \\
\text { Cohen's } d\end{array}$ & $\begin{array}{c}\text { Win } \\
(n=188)\end{array}$ & $\begin{array}{c}\text { Loss } \\
(\mathrm{n}=279)\end{array}$ & $\begin{array}{l}\text { Effect size } \\
\text { Cohen's } d\end{array}$ \\
\hline \multicolumn{8}{|l|}{ Distance } \\
\hline Total distance (TD) & {$[\mathrm{m}]$} & $2817.5 \pm 279.9$ & $2830.9 \pm 313.8$ & 0.04 & $3230.5 \pm 375.5$ & $3172.9 \pm 371.4$ & 0.15 \\
\hline SZ-1: Low velocity running & {$[\mathrm{m}]$} & $2055.8 \pm 203.6$ & $2067.1 \pm 228.2$ & 0.05 & $2188.5 \pm 239.0$ & $2157.1 \pm 240.9$ & 0.13 \\
\hline SZ-2: Crusing & {$[\mathrm{m}]$} & $603.5 \pm 149.0$ & $616.3 \pm 164.6$ & 0.08 & $677.2 \pm 220.9^{*}$ & $666.8 \pm 183.4$ & 0.05 \\
\hline SZ-3: High velocity running & {$[\mathrm{m}]$} & $157.9 \pm 77.6$ & $147.2 \pm 79.9$ & 0.14 & $364.7 \pm 137.4$ & $349.1 \pm 129.6$ & 0.12 \\
\hline \multicolumn{8}{|l|}{ Acceleration } \\
\hline Total acceleration (TA) & [time] & $36.4 \pm 9.8$ & $37.7 \pm 11.1$ & 0.12 & $50.1 \pm 13.1^{*}$ & $51.9 \pm 10.8$ & 0.15 \\
\hline AZ-1: Low acceleration & [time] & $13.9 \pm 4.9$ & $14.1 \pm 5.2$ & 0.03 & $15.4 \pm 5.9$ & $16.2 \pm 5.7$ & 0.15 \\
\hline AZ-2: Moderate acceleration & [time $]$ & $12.2 \pm 4.4$ & $12.4 \pm 4.3$ & 0.05 & $15.4 \pm 5.3$ & $16.1 \pm 5.2$ & 0.13 \\
\hline AZ-3: High acceleration & [time $]$ & $10.3 \pm 4.9$ & $11.3 \pm 5.3$ & 0.18 & $19.4 \pm 6.4$ & $19.6 \pm 5.6$ & 0.04 \\
\hline \multicolumn{8}{|l|}{ Impact } \\
\hline IZ-1: Moderate impact & [time] & $69.5 \pm 45.1^{*}$ & $83.8 \pm 58.0$ & 0.27 & $64.6 \pm 36.8$ & $71.6 \pm 40.4$ & 0.18 \\
\hline IZ-2: High impact & [time] & $20.3 \pm 17.5^{*}$ & $25.2 \pm 24.6$ & 0.22 & $15.8 \pm 13.0^{*}$ & $18.9 \pm 15.3$ & 0.22 \\
\hline
\end{tabular}

Data were presented as mean \pm standard deviation.

Effect sizes of $<0.19,0.20-0.49,0.50-0.79$, and $>0.80$ were considered trival, small, medium, and large, respectively.

* Significant differences $(p<0.05)$ between matches wins and losses.

$\mathrm{TD}=$ total distance; $\mathrm{SZ}=$ speed zone; $\mathrm{TA}=$ total acceleration; $\mathrm{AZ}=$ acceleration zone; $\mathrm{IZ}=$ impact zone. 
Table 2 Impact of score differential on the physical performance in elite japanese rugby union match play.

\begin{tabular}{|c|c|c|c|c|c|c|c|}
\hline & \multirow[b]{2}{*}{ Unit } & \multicolumn{3}{|c|}{ Forwards } & \multicolumn{3}{|c|}{ Backs } \\
\hline & & $\begin{array}{c}\text { Small } \\
(\mathrm{n}=200)\end{array}$ & $\begin{array}{c}\text { Large } \\
(\mathrm{n}=171)\end{array}$ & $\begin{array}{l}\text { Effect size } \\
\text { Cohen's } d\end{array}$ & $\begin{array}{c}\text { Small } \\
(\mathrm{n}=251)\end{array}$ & $\begin{array}{c}\text { Large } \\
(\mathrm{n}=216)\end{array}$ & $\begin{array}{l}\text { Effect size } \\
\text { Cohen's } d\end{array}$ \\
\hline \multicolumn{8}{|l|}{ Distance } \\
\hline Total distance (TD) & {$[\mathrm{m}]$} & $2863.1 \pm 321.8^{*}$ & $2781.3 \pm 266.6$ & 0.27 & $3218.1 \pm 380.6$ & $3170.5 \pm 364.8$ & 0.13 \\
\hline SZ-1: Low velocity running & {$[\mathrm{m}]$} & $2082.0 \pm 240.5$ & $2039.6 \pm 187.1$ & 0.19 & $2193.1 \pm 256.8^{*}$ & $2142.7 \pm 217.2$ & 0.21 \\
\hline SZ-2: Crusing & {$[\mathrm{m}]$} & $624.7 \pm 159.7$ & $595.1 \pm 155.6$ & 0.19 & $671.2 \pm 202.5$ & $670.7 \pm 195.7$ & 0.00 \\
\hline SZ-3: High velocity running & {$[\mathrm{m}]$} & $156.1 \pm 84.8$ & $146.3 \pm 71.6$ & 0.12 & $353.9 \pm 134.1$ & $357.1 \pm 131.8$ & 0.02 \\
\hline \multicolumn{8}{|l|}{ Acceleration } \\
\hline Total acceleration (TA) & [time] & $38.6 \pm 11.3^{*}$ & $35.6 \pm 9.5$ & 0.29 & $51.5 \pm 12.5$ & $50.8 \pm 11.0$ & 0.07 \\
\hline AZ-1: Low acceleration & [time $]$ & $14.6 \pm 5.4^{*}$ & $13.3 \pm 4.6$ & 0.26 & $16.3 \pm 6.3$ & $15.4 \pm 5.1$ & 0.15 \\
\hline AZ-2: Moderate acceleration & [time] & $12.9 \pm 4.5^{*}$ & $11.6 \pm 4.0$ & 0.30 & $15.8 \pm 5.4$ & $15.9 \pm 5.0$ & 0.02 \\
\hline AZ-3: High acceleration & [time] & $11.1 \pm 5.3$ & $10.7 \pm 4.9$ & 0.08 & $19.5 \pm 6.0$ & $19.5 \pm 5.8$ & 0.00 \\
\hline \multicolumn{8}{|l|}{ Impact } \\
\hline IZ-1: Moderate impact & [time] & $80.1 \pm 58.8$ & $75.4 \pm 46.5$ & 0.09 & $67.9 \pm 37.9$ & $69.8 \pm 40.5$ & 0.05 \\
\hline IZ-2: High impact & [time] & $26.3 \pm 24.2^{*}$ & $19.5 \pm 18.7$ & 0.31 & $20.2 \pm 16.0^{*}$ & $14.6 \pm 11.9$ & 0.39 \\
\hline
\end{tabular}

Data were presented as mean \pm standard deviation.

Effect sizes of $<0.19,0.20-0.49,0.50-0.79$, and $>0.80$ were considered trival, small, medium, and large, respectively.

* Significant differences $(p<0.05)$ between matches samll and large.

$\mathrm{TD}=$ total distance; $\mathrm{SZ}=$ speed zone; $\mathrm{TA}=$ total acceleration; $\mathrm{AZ}=$ acceleration zone; $\mathrm{IZ}=$ impact zone.

7 times (win: 65 times; loss: 72 times) were observed in the backs for IZ-1. Differentials of 5 times (win: 25 times; loss: 25 times) and 3 times (win: 16 times; loss: 19 times) for forwards and backs, respectively, were observed for IZ-2. If one is to extrapolate these types of differentials observed in 40 minutes to a full match, an even larger differential is likely to be observed. In addition, forwards endured approximately twice as many impacts as backs. IZ-1 is reported as measuring aspects of the game, such as scrum engagements and tackles, whereas IZ-2 measures aspects, such as tackles and collisions (Cunniffe et al., 2009). IZ-2 also measures breakdowns and collisions with the ground after a tackle. In a losing effort, much time is spent defending against opposition attack, resulting in more tackles being made by the defenders. Thus, impact values are higher in a loss.

In terms of utilizing this information for the strength and conditioning training program, a team with poor results (e.g., a low-ranking team) is more likely to experience wildly fluctuating fortunes in a match during the season and spends a lot of time in defense. Considering this result, there is a need for such team to develop physical fitness that allows for repetitive actions, such as tackles. In addition, the higher impact values in a loss reflect more body contact and an additional physical load. After such match, there is a need to plan for more recovery time. This is particularly true for the forwards, who account for more impacts, and greater weight to this consideration should be afforded them. The method of recovery is detailed. Cunniffe et al. (2010) and Takarada (2003) reports that the higher the number of contacts, the higher the muscle damage (Cunniffe et al., 2010, Takarada, 2003). Therefore, the losing game in which the impact value is high is considered to have high skeletal muscle inflammation. This suggests that a total of 48 hours should be used after the game to control the inflammatory response. As a recovery method, inflammation of the upper body due to contact can be considered; thus, in an ice bath not only the lower body but the whole body is immersed. In the field session, low volume and low intensity sessions should be conducted and drills with contacts should be avoided. Weight training involves adjusting the upper body program.

When comparing performance according to score differential, significantly higher values were found when the score differential was small. Forwards (TD, TA, AZ-1, AZ-2, and IZ-2) and backs (SZ-1 and IZ-2) obtained significantly higher values with small versus large point differentials.

In small differentials, a losing team can overtake the opposition with a single converted try, which makes for a competitive game. In these types of competitive matches when the score differential was small, physical performance parameters were higher. Because players are uncertain how the game will play out, it is believed that their concentration levels and effort/ physical performance are higher. 
In addition, differences in running-related data (TD traveled and TA) were observed among the forwards with different score differentials. In an evenly matched game with a small score differential, players are forced to cover a lot of ground and accelerate repeatedly. For teams faced with such games, it is necessary for players to develop the physical capacity necessary for this type of play. Moreover, forwards should be allowed more recovery time after highly competitive matches as this poses a greater load on the skeletal muscles of the lower extremities.

Furthermore, IZ-2 showed significantly higher values in both positions when the score differential was small. It is thought that the higher values reflect the faster defensive responses necessary to defend against the opposition attack. These results can provide important information to coaches when making player selections and considering substitutions according to the progression of a game. In closely fought matches, coaches can select forwards with more endurance and/ or substitute players in these positions earlier than usual.

From the results of this study, both forwards and backs showed the highest physical performance for each parameter when the score differential was small. The maximum values per 40 minutes for each forwards and backs were found, and this result can be applied to training and rehabilitation programs. In field training, by implementing programs that reflect the results obtained, it is possible to adapt to severe match that require the highest performance. By building strength and fitness that can adapt to the toughest matches, it will be possible to respond to any match. Moreover, by developing a rehabilitation program based on the maximum value of physical performance, it is possible to prevent reinjury after returning to the competition. Thus, the results obtained in this study could be used as reference values for objective evaluation in training and rehabilitation.

In previous research about rugby league, physical performance values were higher in a win or large score differential (Gabbett, 2013). The results of this study about rugby union contradict those results; however, a previous study on Australian Rules football has shown some trends toward similar results to this study (Sullivan et al., 2014). It is thought that this could be a result of differences in the rules between rugby league and rugby union. Although physical performance in every sport is influenced by match results, the impact differs with the sport. Therefore, different training methods will be needed for improving performance. In all cases, understanding the relationship between physical performance and match results could be significant in improving performance and preventing sports injuries in future competitions.

As backs exhibited significantly higher TA values in a loss, backs is a position where high volume and speed \& agility are required mainly during a matc. But in case of a loss or when the score differential was small, the demand for impact increases like forwards. To win a severe match, backs must work on tackle improvement. In addition, we believe that more detailed information can be obtained by analyzing each position (Scrum half, Fly half, Centres, Wingers, Full back) that constitutes the backs.

In this study, only one team belonging to the top league participated. Physical performance may also be affected by team tactics. Therefore, it is necessary to further examine the relationship between physical performance and game development for multiple teams.

\section{Conclusion}

The purpose of this study was, to clarify the impact of match results (win-loss, winning margin) on physical performances in rugby union teams. In addition, it was to provide ideas reflecting the findings obtained from this study on the pitch.

From the result of this study, match results (winloss, winning margin) had an effect on physical performance. In particular, each parameter returned high values in closely fought matches when a team lost or when the points differential was small. In addition, it is clear that physical performance is impacted differently from one position to another. Suggestions for new approaches that can be adopted by strength and conditioning coaches in training in order to improve performance and to prevent sports injuries have been noted. Furthermore, it has been mentioned that the findings from this research can be used by coaches when selecting and substituting players.

\section{Conflict of Interest}

The authors declare no conflict of interest associated with this work. This research did not receive any specific grant from funding agencies in the public, commercial, or not-for-profit sectors.

\section{Acknowledgments}

The authors would like to thank Dr. Takemura for his expert statistical help and Enago (www.enago.com) for the English language review. 


\section{References}

Austin, D., Gabbett, T., and Jenkins, D. (2011). The physical demands of Super 14 rugby union. J. Sci. Med. Sport, 14: 259263.

Cunniffe, B., Hore, A. J., Whitcombe, D. M., Jones, K. P., Baker, J. S., and Davies, B. (2010). Time course of changes in immuneoendocrine markers following an international rugby game. Eur. J. Appl. Physiol., 108-113.

Cunniffe, B., Proctor, W., Baker, J. S., and Davies, B. (2009). An evaluation of the physiological demands of elite rugby union using Global Positioning System tracking software. J. Strength Cond. Res., 23: 1195-1203.

Duthie, G., Pyne, D., and Hooper, S. (2003). Applied physiology and game analysis of rugby union. Sports Med., 33: 973-991.

Fuller, C. W., Brooks, J. H., Cancea, R. J., Hall, J., and Kemp, S. P. (2007). Contact events in rugby union and their propensity to cause injury. Br. J. Sports Med., 41: 862-867.

Gabbett, T. J. (2013). Influence of the opposing team on the physical demands of elite rugby league match play. J. Strength Cond. Res., 27: 1629-1635.

Jennings, D., Cormack, S. J., Coutts, A. J., and Aughey, R. J. (2012). GPS analysis of an international field hockey tournament. Int. J. Sports Physiol. Perform., 7: 224-231.

Kempton, T., Sirotic, A. C., and Coutts, A. J. (2014). Between match variation in professional rugby league competition. J. Sci. Med. Sport, 17: 404-407.

Mallo, J., Mena, E., Nevado, F., and Paredes, V. (2015). Physical demands of top-class soccer friendly matches in relation to a playing position using global positioning system technology. J. human kinetics, 47: 179-188.

McLaren, S. J., Weston, M., Smith, A., Cramb, R., and Portas, M. D. (2016). Variability of physical performance and player match loads in professional rugby union. J. Sci. Med. Sport, 19: 493-497.

Quarrie, K. L., Hopkins, W. G., Anthony, M. J., and Gill, N. D. (2013). Positional demands of international rugby union: Evaluation of player actions and movements. J. Sci. Med. Sport, 16: 353-359.

Rampinini, E., Coutts, A. J., Castagna, C., Sassi, R., and Impellizzeri, F. (2007). Variation in top level soccer match performance. Int. J. Sports Med., 28: 1018-1024.

Reilly, B., Akubat, I., Lyons, M., and Collins, D. K. (2015). Match-play demands of elite youth Gaelic football using global positioning system tracking. J. Strength Cond. Res., 29: 989996.

Sullivan, C., Bilsborough, J. C., Cianciosi, M., Hocking, J., Cordy, J., and Coutts, A. J. (2014). Match score affects activity profile and skill performance in professional Australian Football players. J. Sci. Med. Sport, 17: 326-331.

Takarada, Y. (2003). Evaluation of muscle damage after a rugby match with special reference to tackle plays. Br. J. Sports Med., 37: 416-419.

Wisbey, B., Montgomery, P. G., Pyne, D. B., and Rattray, B. (2010). Quantifying movement demands of AFL football using GPS tracking. J. Sci. Med. Sport, 13: 531-536.

Yamamoto, H., Takemura, M., Iguchi, J., Tachibana, M., Tsujita, J., and Hojo, T. (2020). In-match physical demands on elite Japanese rugby union players using a global positioning system. BMJ Open Sport Exer. Med., 6: 1-10.

Yamamoto, H., Takemura, M., Kaya, M., and Tsujita, J. (2017). Physical demands of elite rugby union match-play using global positioning system. Football Sci., 14: 15-23.

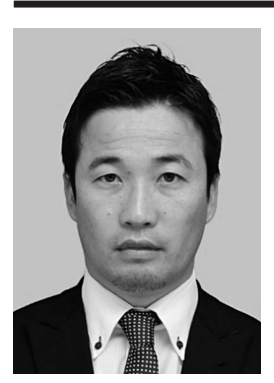

Name:

Hayato Yamamoto

\section{Affiliation:}

Graduate School of Health and Sports Science, Doshisha University

\section{Address:}

1-3 Tataramiyakodani, Kyotanabe, Kyoto 610-0394 Japan

Brief Biographical History:

2013- present Head Strength and Conditioning Coach, NTTdocomo Rugby Football Club RedHurricanes

2015-2016 Strength and Conditioning Coach, Japan national rugby sevens team

2016-2020 Doctoral course, Graduate School of Health and Sports Science, Doshisha University

2019- present Strength and Conditioning Coordinator, Posco Rugby Football Club (Korea)

\section{Main Works:}

-Yamamoto, H., Takemura, M., Kaya, M., and Tsujita, J. (2017). Physical demands of elite rugby union match-play using global positioning system. Football Sci., 14: 15-23.

-Yamamoto, H., Takemura, M., Iguchi, J., Tachibana, M., Tsujita, J., and Hojo, T. (2020). In-match physical demands on elite Japanese rugby union players using a global positioning system. BMJ Open Sport Exer. Med., 6: 1-10.

Membership in Learned Societies:

- National Strength and Conditioning Association Japan

- Japan Association of Training Instructors

- Japanese Society of Physical Fitness and Sports Medicine

- American College of Sports Medicine

- Japanese Society of Science and Football

-European College of Sport Science 